\title{
Excess coronary artery disease risk in South Asian immigrants: Can dysfunctional high-density lipoprotein explain increased risk?
}

\author{
Sunita Dodani \\ Medical College of Georgia, \\ Augusta, GA, USA
}

Correspondence: Sunita Dodani Room 4503, Health Sciences building, Medical College of Georgia, 997 St. Sebastian Way, Augusta, GA, 30912, USA

Tel + I 7067210426

Fax +I 7067217049

Email sdodani@mcg.edu
Background: Coronary artery disease (CAD) is the leading cause of mortality and morbidity in the United States (US), and South Asian immigrants (SAIs) have a higher risk of CAD compared to Caucasians. Traditional risk factors may not completely explain high risk, and some of the unknown risk factors need to be explored. This short review is mainly focused on the possible role of dysfunctional high-density lipoprotein (HDL) in causing CAD and presents an overview of available literature on dysfunctional HDL.

Discussion: The conventional risk factors, insulin resistance parameters, and metabolic syndrome, although important in predicting CAD risk, may not sufficiently predict risk in SAIs. HDL has antioxidant, antiinflammatory, and antithrombotic properties that contribute to its function as an antiatherogenic agent. Recent Caucasian studies have shown HDL is not only ineffective as an antioxidant but, paradoxically, appears to be prooxidant, and has been found to be associated with CAD. Several causes have been hypothesized for HDL to become dysfunctional, including Apo lipoprotein A-I (Apo A-I) polymorphisms. New risk factors and markers like dysfunctional HDL and genetic polymorphisms may be associated with CAD.

Conclusions: More research is required in SAIs to explore associations with CAD and to enhance early detection and prevention of CAD in this high risk group.

Keywords: South Asian immigrants, coronary heart disease, cardiovascular risk, high-density lipoprotein

\section{Coronary artery disease in South Asians: what is known and what remains to be known}

Among cardiovascular diseases, coronary artery disease (CAD) is the leading cause of death in the United States (US), and certain populations, such as South Asians, African Americans, and Hispanics, carry a disproportionately larger burden of CAD (Uppaluri 2002; Nordlie et al 2005; Joshi et al 2007). The mortality rates from CAD in South Asians are reported to be two to three times higher than those for Caucasians, irrespective of gender, religion, social class, dietary practices or country of residence (Enas et al 1992; Joshi et al 2007). This higher prevalence is seen in South Asian immigrants (SAIs) in the US as well as in those living in India, and rates are similar among vegetarians and nonvegetarians (Enas et al 1992, 1996; Mohan et al 2001; Goyal and Yusuf 2006; Joshi et al 2007). South Asian Indian women in California have the highest death rate from CAD in the United States, higher than Caucasians, Blacks, Hispanics, and Native Americans (Klastsy et al 1994). Specifically, 33\% of all deaths among Indian women are due to CAD compared with $11 \%$ among Japanese, 16\% among Chinese, and 20\% among Hispanics, Blacks, and Caucasians. In California, SAIs have the highest rate of hospitalization for CAD, and Chinese have the lowest rate. The high rates of CAD among SAIs are not limited to the US and appear 
to be part of a global phenomenon (Bhopal 2002). Further, CAD risk factors are present in South Asians at a younger age compared to other populations, resulting in CAD at a younger age than in other populations (joshi et al 2007). The reasons for early development of CAD risk factors at a relatively young age in SAIs are still unclear.

More than 3.6 million South Asians live in the US, and although this group represents the second fastest growing Asian immigrant population, little is known regarding their increased risk for CAD (Uppaluri 2002). Because the population is scattered across the nation, it has been difficult to fully study the problem and to raise awareness within communities. There has been much speculation about what causes the increased occurrence of and mortality from CAD in SAIs. Not uniform groups, South Asians include ethnic subgroups with different cultures and practices, and the prevalence of recognized risk factors for CAD varies among the subgroups (Anand et al 2000; Bhopal 2002; Bhatnagar et al 2005; Goyal and Yusuf 2006). As a whole, however, South Asians and SAIs have a much higher prevalence of diabetes, insulin resistance, central obesity, dyslipidemias (lower high-density lipoprotein [HDL], increased lipoprotein [a], higher triglycerides), increased thrombotic tendency (increased plasminogen activator inhibitor-1 and decreased tissue plasminogen activator levels), decreased levels of physical activity, and lower birth weights, commonly known as "fetal origins hypothesis syndrome" (Forouhi and McKeigue 1997; Anand et al 2000; Grundy 2002; Bhatnagar et al 2005; Goyal and Yusuf 2006; Joshi et al 2007).

The higher CAD risk in South Asians may be related to a higher prevalence of metabolic syndrome which has become increasingly common among SAIs (Grundy 2002; Forouhi and McKeigue 1997; Forouhi and Sattar 2006) as compared to other populations (Figure 1). The syndrome is characterized by increased abdominal obesity, atherogenic dyslipidemias, high blood pressure, insulin resistance, glucose intolerance, prothrombotic state, and pro-inflammatory state. However, even taking these differences into account, classical risk factors may not fully explain the increased risk for CAD in South Asians (Forouhi and McKeigue 1997; Grundy 2002; Forouhi and Sattar 2006; Joshi et al 2007).

The CAD rates in the US, Australia, Canada, France, Japan, and Finland (Peterson et al 2004) have declined to half over the past 30 years. These vast reductions in CAD mortality are generally attributed to nationwide changes in specific risk factors that were identified through epidemiological research and addressed through population-based interventions (McGovern et al 1996; Luepker et al 2006; Kottke et al 2006). Reduction in risk factors explains most of the decline with modest contributions from advances in treatment (SSSG 1994; Shepherd et al 1995; Sacks et al 1996; Downs et al 1998; HPS 2002). Ironically, during this time, the CAD rates doubled in SAIs, and the impact of risk factors and genetic susceptibility appears to be greater in this population compared with others, making SAIs a high-risk group (Enas 2000). Migration is an important factor in determining the increased risk of CAD; however, other migrating populations (eg, Afro-Caribbean) do not have an increased risk of CAD compared with the indigenous population (Williams 1995). Thus, other specific factors must apply to SAIs.

\section{Dyslipidemias in South Asians}

Among numerous genetic and lifestyle parameters, dyslipidemias are one of the most prominent risk factors for CAD. Epidemiological studies have identified low-density lipoprotein (LDL) and HDL as independent risk factors that modulate CAD risk (Gordon et al 1989). Though the primary

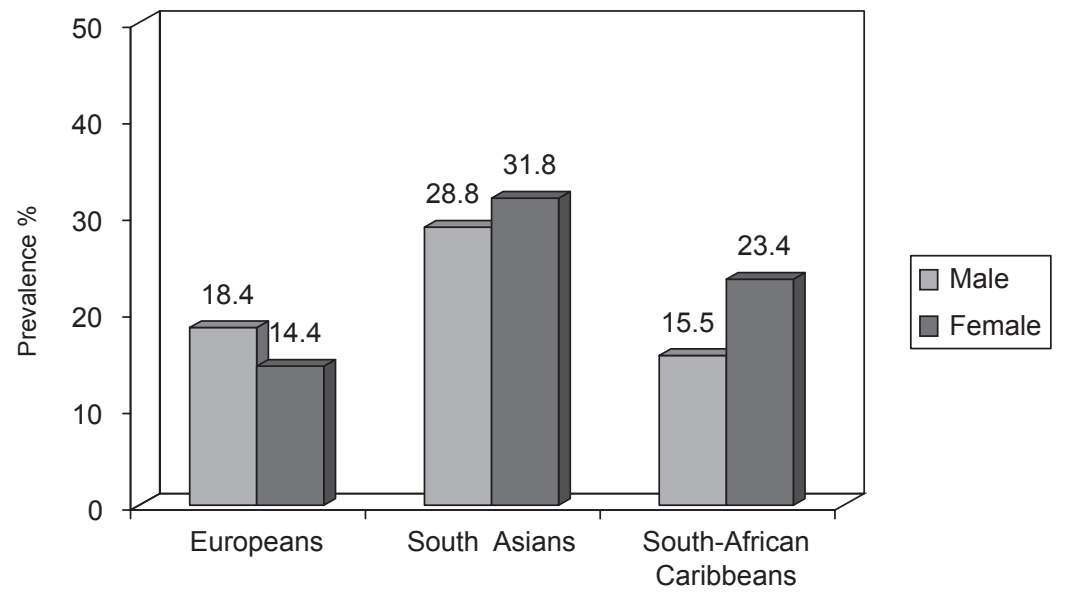

Figure I Age-standardized prevalence of metabolic syndrome defined by NCEP ATP II criteria. 
goal is to reduce LDL levels, however HDL cholesterol levels are among the most predictive risk factors for CAD (Gordon et al 1989; Tulenko and Sumner 2002). A growing body of experimental evidence suggests that augmenting the levels or function of HDL and its apolipoproteins can have major vascular protective effects ranging from prevention to stabilization and regression, independent of total or nonHDL cholesterol levels (SSSG 1994; Shephered et al 1995; Sacks et al 1996; Downs et al 1998; HPS 2002). Recent clinical trials have stimulated tremendous interest in the structure, function, and therapeutic potentials of HDL. The National Cholesterol Educational Program Adult Treatment Panel III (NCEP ATP III) guidelines clearly define an HDL level of $<40 \mathrm{mg} / \mathrm{dl}$ as an independent risk factor for CAD, and low HDL is often present in high-risk patients with CAD (NCEP ATP III 2001). Although an inverse relationship between HDL and risk of acute myocardial infarction is highly significant, the relationship is far from perfect.

The common pattern of dyslipidemias seen in SAIs relative to other populations is shown in Table 1 (Anand et al 2003). SAIs tend to have higher triglyceride levels, lower HDL levels, and higher lipoprotein a (Lpa) levels. However, a few recent studies on SAIs in the US showed that the average HDL level in both men and women was either normal or high (Hoogeveen et al 2001; Kalhan et al 2001). Whether this difference in HDL level is due to differences in migration, physical activity, gene-environment interactions, or other unknown factors is not known.

Based on known ethnic differences in risk prediction, the Framingham prediction model accurately predicts the CAD risk among Caucasians and blacks living in the United States. For Americans of Japanese and Hispanic descent and for Native Americans, the Framingham model overestimates CAD risk. For SAIs, the Framingham model underestimates CAD risk by greater than $100 \%$ (D'Agostino et al 2001; Bhopal et al 2005; Forouhi and Sattar 2006). Total cholesterol and LDL levels are correlated with the extent and severity of CAD in SAIs as in Caucasians, but at any given total cholesterol or LDL level, SAIs have a greater CAD risk than Caucasians (D'Agostino et al 2001; Bhopal et al 2005; Forouhi and Sattar 2006). Therefore, SAIs with dyslipidemias should be treated as the same level of importance as if they had a CAD risk equivalent to patients with diabetes or heart disease.

Over the past decade, lowering LDL levels has been the major target in cardiovascular protection strategies and clinical trials have clearly established that reductions in LDL are associated with a $30 \%-45 \%$ reduction in clinical events (SSSG 1994; Shepherd et al 1995; Sacks et al 1996; Downs et al 1998; HPS 2002). However, despite low LDL and normal HDL levels, many patients continue to have cardiac events. One can calculate from the published data (Castelli et al 1986) that $44 \%$ of the CAD clinical events occurred in men with HDL levels greater than $40 \mathrm{mg} / \mathrm{dl}$ and $43 \%$ in women with HDL levels greater than $50 \mathrm{mg} / \mathrm{dl}$. Because a significant number of CAD events occur in patients with normal LDL and HDL levels, there has been a continuing search for markers with better predictive value.

\section{HDL role in CAD}

Lipoproteins are complexes of proteins, phospholipids, and cholesterol. HDL is the smallest and densest of the lipoproteins because of its high protein content. Proteins make up about $50 \%$ of its mass and apolipoprotein A-I (Apo A-I) accounts for $70 \%$ of that protein component (Gordon 1989).

Apo A-I is a 243 amino acid protein synthesized in the liver $(70 \%)$ and intestine $(30 \%)$ and secreted into the serum in a lipid-free state (Tulenko and Sumner 2002). Individual Apo A-I molecules associate with other Apo A-I molecules, membrane phospholipids, and cholesterol to form lipid-poor pre- $\beta$-HDL. Lipid-poor HDL assumes a double-belt-like structure which becomes spherical with maturation and addition of cholesterol (Tulenko and Sumner 2002).

Lipid-poor $\beta$-HDL is able to take up cholesterol from artery wall macrophages via the ATP-binding cassette A-1

Table I Dyslipidemias in South Asian immigrants relative to other populations (Anand et al 2000)

\begin{tabular}{|c|c|c|c|c|}
\hline Factor & South Asian & †European & Chinese & ${ }^{\dagger} \mathbf{p}$ \\
\hline Total cholesterol (mg/dl) & $211.77(81.90)$ & I84.86 (8I.90) & $183.69(81.90)$ & 0.0001 \\
\hline LDL (mg/dl) & $128.70(35.10)$ & $123.63(31.20)$ & $122.46(31.20)$ & 0.03 \\
\hline HDL (mg/dl) & $38.56(11.70)$ & 46.41 (II.70) & 46.41 (II.70) & 0.0001 \\
\hline Triglycerides (mg/dl) & $174.44(\mid 15.70)$ & $145.96(\mid 15.70)$ & I58.42 (I I5.70) & 0.005 \\
\hline Lipoprotein (a) $(\mathrm{g} / \mathrm{L})^{\ddagger}$ & $0.0293(0.018)$ & $0.0259(0.0119)$ & $0.0259(0.0119)$ & 0.001 \\
\hline
\end{tabular}

Abbreviations: LDL, low-density lipoprotein; HDL, high-density lipoprotein. Notes: ${ }^{\dagger}$ mean (SD), ${ }^{\ddagger}$ Natural Log. 
(ABCA-1) receptor (Gordon 1989). Cholesterol is transferred into lipid-poor HDL and is then esterified by the enzyme lecithin-cholesterol acyltransferase. As cholesterol is esterified it is packaged into the core of the HDL leading to formation of the mature spherical lipoprotein.

Mature spherical HDL can then unload its cholesterol by 2 main mechanisms. Transfer of cholesterol back to the liver is facilitated by interaction with the scavenger receptor-B1 (SR-B1) receptor on hepatocytes and leads to formation of bile and secretion into the gut. Alternatively, cholesterol can be transferred from mature HDL to LDL or very-low-density lipoprotein (VLDL), a process dependent on cholesteryl ester transfer protein (CETP). This leads to a recycling of cholesterol, potentially back into the artery wall (Gordon 1989; Tulenko and Sumner 2002).

HDL has antioxidant, antiinflammatory, and antithrombotic properties that contribute to its function as an antiatherogenic agent. Although our understanding of how HDL protects against CAD is still incomplete, evidence supports at least three major athero-protective mechanisms of HDL.

1. HDL is an integral component of the reverse cholesterol transport process, functioning as a carrier of excess cellular cholesterol from peripheral tissues to the liver, where it is excreted from the body as bile acids and cholesterol. More specifically, HDL mediates efflux of cholesterol from cholesterol-loaded macrophages by passive diffusion, through scavenger receptor B1 (SR B1), and, most significantly, via the protein Apo A-I as mentioned above (Williams et al 1999; Yancey et al 2003). Apo A-I functions through an ATP-binding cassette transporter A1 (ABCA1) in the vessel wall, where it accepts free cholesterol, forming pre-beta HDL that matures after esterification to cholesteryl esters (CE) and then by lecithin-cholesterol acyltransferase to alpha migrating HDL (Takahashi and Smith 1999; Remaley et al 2000; Mackness et al 2002).

2. A series of antioxidant enzymes which protect LDL from oxidation are associated with HDL. Oxidized lipids are transferred to HDL from LDL and are hydrolyzed by HDL-associated PON1, LCAT, and platelet-activating factor (PAF) acetylhydrolase enzymes (Navab et al 2001; Mackness et al 2002; Marathe et al 2002). The activities of these enzymes destroy oxidized lipids and also inhibit their formation. Removing the oxidized lipids initiates a positive feedback loop that results in further activation of the enzymes and further destruction of the oxidized lipids. In addition, Apo A-I reduces lipid peroxides within LDL, independent of PON1 (Marathe et al 2002).
3. HDL may protect against CAD by selectively decreasing endothelial cell adhesion molecules, which facilitate the binding of mononuclear cells to the vessel wall and promote lesion development (Barter et al 2002).

\section{Evidence that HDL may not be protective: dysfunctional HDL}

According to several recent studies, in patients with CAD, HDL is not only ineffective as an antioxidant but, paradoxically, appears to be pro-oxidant, promoting LDL oxidation and monocytes chemotactic activity in the human artery wall as well as increasing HDL lipid hydroperoxides, as assessed by its lipid peroxide content (Navab et al 2000; Fogelman 2004; Ansell et al 2005, 2006). In addition, HDL from patients with a history of CAD enhances the oxidation of LDL and of phospholipids in LDL (Ansell et al 2006; Navab et al 2006). This pro-inflammatory HDL, which is dysfunctional, accumulates oxidants that inhibit HDL-associated antioxidant enzymes, render Apo A-I unable to promote ABCA1 mediated cholesterol efflux, and promotes the formation of LDLderived oxidized lipids (Fogelman 2004) (Figure 2). The mechanisms underlying this phenomenon are not completely understood. One of the hypothesized mechanisms involves myeloperoxidase (Zheng et al 2004). Apo A-I is targeted by myeloperoxidase, and, when oxidized and nitrated, impairs ABCA1-dependent cholesterol efflux. Furthermore, exposing HDL or Apo A-I to myeloperoxidase almost entirely prevents ABCA1-dependent reverse cholesterol transport (Fogelman 2004). It has been hypothesized that a certain variant of Apo A-I is susceptible to oxidation and nitration (Navab et al 2004).

The diagnosis of dysfunctional HDL has historically been made with a cell-based assay that requires endothelial cells, smooth muscle cells, and monocytes. However, the use of a cell-based assay is not practical for large-scale studies. Recently, a cell-free assay was developed to detect HDL that is dysfunctional in preventing the formation or inactivation of oxidized phospholipids (Navab et al 2001; Ansell et al 2003, 2007). This is a rapid test for HDL function that does not require cells and gives results highly comparable to those of the previously described cell-based assay (Navab et al 2001). Using this assay, investigators determined that HDL from patients with documented CAD was pro-inflammatory (dysfunctional), while HDL from normal subjects was antiinflammatory. These patients had normal levels of blood lipids, had no major risk factors for CAD, and therefore, would not have been predicted to be at risk for atherosclerosis by conventional risk factor analysis. In short, the new cell-free 


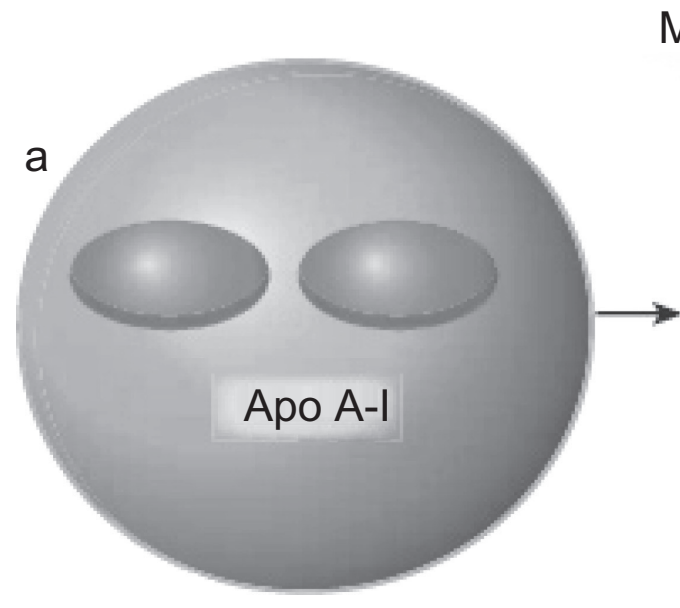

Anti-inflammatory

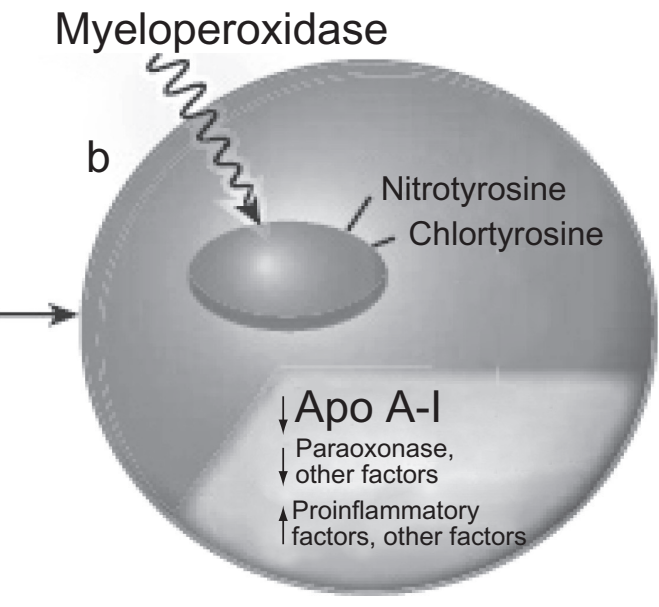

Proinflammatory

Figure 2 Model of bidirectional conversion of high-density lipoprotein from antiinflammatory (a) to proinflammatory (b). Copyright (c) 2004. Reproduced with permission from Fogelman AM. 2004. When good cholesterol goes bad. Nat Med, 10:902-3.

Abbreviation: Apo A-I, apolipoprotein A-I.

assay has the potential to allow wide-spread testing for HDL that is dysfunctional and identification of individuals at risk for CAD.

The inflammatory/antiinflammatory properties of HDL can be determined by calculating HDL inflammatory index (Navab et al 2001; Ansell et al 2003, 2007). This index is calculated by normalizing the cell-free assay values obtained for a standard LDL alone to $<1.0$. Using this normalization procedure, a test HDL is classified as proinflammatory (dysfunctional) when addition of the test HDL along with an LDL standard to the assay results in a normalized value of 1.0 or greater. Conversely, a value less than 1.0 classifies the test HDL as antiinflammatory. A small study of 20 Caucasian subjects with elevated HDL showed an inflammatory index of $1.28 \pm 0.29$ in CAD patients compared to $0.35 \pm 0.29$ in healthy subjects, clearly separating patients from controls (Ansel et al 2003).

\section{Proinflammatory (dysfunctional) HDL in SAls?}

Current data indicates that a $1 \%$ increase in HDL serum concentration can decrease cardiovascular risk by $2 \%-3 \%$, independent of LDL levels (Gordon et al 1989). However, HDL can have this protective effect only if it is functional. The incidence of prooxidant or proinflammatory HDL (dysfunctional HDL) in SAIs is not known.

A small study done on SAIs showed inflammatory index values of $\geq 1.00$ in $50 \%$ (95\% CI $0.8772-1.4333$ ) with normal HDL suggesting proinflammatory or dysfunctional
HDL (Dodani et al 2008b). In addition, proinflammatory HDL ( $\geq 1.00)$ was associated with CAD, using IMT values $\geq 0.80 \mathrm{~mm}$ as surrogate marker for CAD ( $\mathrm{p}=0.04$ ). The mean HDL inflammatory Index was 0.8772 in the group with CAD and 0.6269 in the group without CAD. Further work is needed to understand the relationship of dysfunctional HDL with HDL levels.

Given that animal and small-scale human studies suggest that measures of the quality and functionality of HDL can provide an improved means of identifying subjects at increased risk for atherosclerotic events, compared with the current practice of only measuring the level of HDL (NCEP ATP III 2001), the level of dysfunctional HDL and its association with CAD in SAIs needs to be explored in bigger studies. Given the results of small studies, we can hypothesize that SAIs have a high prevalence of dysfunctional HDL and that this could be related to their excessive risk of CAD. We are currently funded by the National Institute of Health (NIH) to study association of dysfunctional HDL with CAD in SAIs. Similarly, SAIs carry an increased prevalence of metabolic syndrome and diabetes (subclinical chronic inflammation) as compared to other groups, therefore, we predict that this population has increased dysfunctional HDL and is related to coronary events (Grundy 2002; Tillin et al 2005; Forouhi and Sattar 2006). Patients with metabolic syndrome tend to have dysfunctional HDL (proinflammatory HDL) more often than controls (Watson et al 2007). Identification of dysfunctional HDL and its association with carotid IMT and 
metabolic syndrome in SAIs will help to identify high-risk individuals; high risk that cannot be explained by traditional risk factors. Patients found to have dysfunctional HDL and no other CAD risk factors can be targeted for early lipidlowering treatment which has been shown to improve HDL antiinflammatory function (Ansell et al 2003, 2007; Kumar and Reynolds 2005). However, weather measures of dysfunctional HDL are superior to other indirect markers like total cholesterol/HDL ratio, Apolipoprotein B/Apo A-I ratio requires more research.

\section{Apo A-I and gene polymorphisms}

Apo A-I (APOAI gene, Apo A-I protein) is the major protein of HDL and consists of a 243 amino acid-long peptide, synthesized mainly in the liver and to some extent in the small intestine. The inverse relationship between HDL plasma levels and CAD has been attributed to the role that HDL and its major constituent Apo A-I play in reverse cholesterol transport (RCT). The efficiency of RCT depends on the specific ability of Apo A-I to promote cellular cholesterol efflux, bind lipids, activate lecithin: cholesterol acyltransferase (LCAT), and form mature HDL that interacts with specific receptors and lipid transfer proteins (Stampfer et al 1991; Karathanasis et al 1985; Frank and Marcel 2000). The APOAI gene is present along with the APOC3 and APOA4 genes, on chromosome 11(11q23.3-qter). It has also been shown that the A allele of the APOAI gene contributes to the severity of CAD and low levels of HDL among Northern Indians (Chhabra et al 2005).

Epidemiological studies have shown that HDL and Apo A-I levels are inversely correlated with the risk of developing CAD (Jadhav and Kadam 2004; Navab et al 2005; Chhabra et al 2005). Although various factors such as genetic variations, diet, exercise, alcohol, smoking, hormones, and certain drugs can significantly influence the levels of HDL and Apo A-I (Lusis et al 2004), family and twin studies have demonstrated a strong genetic heritability, accounting for up to $66 \%$ of the variability of HDL and Apo A-I levels (Calbresi et al 1997; Jadhav and Kadam 2004; Chhabra et al 2005). Furthermore, $40 \%-60 \%$ of the inter-individual variation in HDL concentrations is controlled at the genetic level (Chhabra et al 2005) and the strong positive correlation between plasma levels of Apo A-I and HDL suggests that Apo A-I gene polymorphisms may be linked to variability in HDL levels as well as to dysfunction (Chhabra et al 2005; Navab et al 2005).

More than 40 mutations in Apo A-I have been identified in several ethnic populations (Moll et al 1989;
Koistinen et al 1994; Calabresi and Franceschini 1997; Jadhav and Kadam 2004; Lusis et al 2004; Chhabra et al 2005), each producing differing HDL activity and levels. Apo A-I gene mutations are separated into three classes. Class I includes mutations that prevent the formation of Apo A-I; class II defects lead to expression of a truncated Apo A-I protein; and class III defects result in an altered functional state of Apo A-I. Not surprisingly, class I mutations are associated with CAD, xanthomatosis, and corneal opacifications, whereas class II mutations exhibit variable phenotypes (Moll et al 1989; Koistinen et al 1994; Calabresi and Franceschini 1997; Frank and Marcel 2000; Lusis et al 2000). Mutations affecting the structure of Apo A-I (nonsense mutations with premature stop codons or large genomic deletions) result in an absence of Apo A-I in plasma and a marked reduction in HDL levels (Moll et al 1989). Interestingly, of the 12 mutations that truncate Apo A-I, not all are associated with CAD. Further, several point mutations have been identified (Moll et al 1989; Koistinen et al 1994; Calabresi and Franceschini 1997; Frank and Marcel 2000), and one in particular is associated with low levels of HDL but not with an increased CAD risk and is, instead, associated with a reduction in CAD risk (Boekholdt et al 2006). This Apo A-I mutation is called Apo A-I milano (Apo A-I $\mathrm{Arg}_{173 \mathrm{Cys}}$ ).

\section{Apo A-I polymorphisms in SAls}

A majority of the studies on Apo A-I polymorphisms and their association with HDL level and CAD have been carried out in Caucasian and other populations. The association of Apo A-I gene polymorphisms with carotid IMT as a surrogate marker for atherosclerosis has been examined, but not fully. For example, Apo A-I (L178P) was found to be associated with high IMT measurements $(p<0.001)$ in a European population, however due to the small sample size, results can not be generalized (Pulkkinen et al 2000). Similarly, Apo A-I/C-III/A-IV SstI polymorphism was found to be associated with high carotid IMT measurements in a study of a young Finnish population (Wang et al 1996). However, the role of Apo A-I polymorphisms in individuals with metabolic syndrome and its association with dysfunctional HDL has not yet been fully studied. A few studies have examined Apo A-I polymorphisms in native populations of South Asians from India, however, to our knowledge, no study has examined Apo A-I polymorphisms in SAIs and its association with dysfunctional HDL, IMT, or CAD. Polymorphisms in Apo A-I and other lipid metabolismrelated genes have been suggested to be clinically useful in assessing an individual's risk for cardiovascular disease 
and in conducting genetic-epidemiological evaluations (Perez-Mendez et al 2000).

Given that SAIs are significantly under-represented in major clinical trials, evidence-based management strategies for treatment and prevention of CAD specifically in this population is seriously lacking. A literature search for Apo A-I gene mutations in South Asian populations yielded a small study that showed polymorphisms in the Apo lipoprotein C-III promoter gene that were associated with features of metabolic syndrome in South Asian Indians; the relationship of these polymorphisms to CAD was not examined (Hovingh et al 2004; Guettier 2005; Islam et al 2005). A recent small study conducted on Pakistanis suggested that the promoter region of the Apo A-I gene may play a role in determining blood pressure (Sadaf et al 2002), however, due to insufficient power, these results cannot be generalized. Chhabra and colleagues (2005) found a correlation between the expression of the Apo A-I G-75A polymorphism in northern Indians, the severity of CAD, and low levels of HDL; however this study was restricted to one ethnic group and relationship was not examined in other ethnic groups. Studies have shown that South Indians carry more CAD risk as compared to North Indians (Hoogeveen 2001).

In a small study on SAIs, six novel polymorphisms were identified, one of which, G4 (C938T), was significantly associated with low $(<40 \mathrm{mg} / \mathrm{dl})$ HDL levels $(\mathrm{p}=0.03)$ (Dodani et al 2008a). Further research is required to explore Apo A-I polymorphisms in SAIs and correlate possible associations with dysfunctional HDL and CAD. Moreover, it may lead to screening tests that will allow early detection and control of the developing CAD. Also these tests may lead to the development of gene therapy mechanisms useful in the treatment of CAD in SAIs.

\section{Conclusion}

People of South Asian origin constitute a large, visible minority in the United States and are known to be at heightened risk for premature CAD. Conventional risk factors clearly confer risk in South Asians but do not adequately explain their excess risk compared with other populations. New risk factors and markers like dysfunctional HDL, genetic polymorphism though shown to be linked with CAD; however greater research is required in South Asians. The rates of CAD have accelerated dramatically amongst South Asians, driven to an important extent by the atherogenic dyslipidemia and type 2 diabetes that have become so common amongst them. South Asians may have a genetic predisposition to CAD; however, environmental, nutritional, and lifestyle factors may also be responsible. South Asians have a much higher prevalence of metabolic syndrome, diabetes, insulin resistance (and resultant hyperinsulinemia), central obesity, dyslipidemias (lower HDL, increased lipoprotein[a], higher triglyceride levels), increased thrombotic tendency (increased plasminogen activator inhibitor-1 and decreased tissue plasminogen activator levels), decreased levels of physical activity, and low birth weights ("fetal origins hypothesis"). In addition, the dietary indiscretions and sedentary lifestyle practiced by most South Asians puts them at a higher risk. A multidisciplinary approach involving the population at risk, healthcare personnel, and the government is required to diminish the incidence.

The key to combating the increasing incidence of CAD among South Asians is the treatment of known risk factors through both an individual-based as well as a populationbased approach aimed at comprehensive risk factor reduction. In addition, further research is needed to discover new risk factors like dysfunctional HDL that can be responsible for an excess CAD risk, not explained by conventional risk factors.

\section{Disclosure}

The author reports no conflicts of interest in this work.

\section{References}

Anand SS, Yusuf S, Vuksan V, et al. 2000. Differences in risk factors, atherosclerosis, and cardiovascular disease between ethnic groups in Canada: the Study of Health Assessment and Risk in Ethnic groups (SHARE). Lancet, 356:279-84.

Ansell BJ, Fonarow GC, Fogelman AM. 2005. High-density lipoprotein function recent advances. J Am Coll Cardiol, 46:1792-8.

Ansell BJ, Fonarow GC, Fogelman AM. 2006. High-density lipoprotein: is it always atheroprotective? Curr Atheroscler Rep, 8:405-11.

Ansell BJ, Fonarow GC, Navab M, et al. 2007. Modifying the anti-inflammatory effects of high-density lipoprotein. Curr Atheroscler Rep, 9:57-63.

Ansell BJ, Navab N, Hama S. 2003. Inflammatory/anti-inflammatory properties of high-density lipoprotein distinguish patients from control subjects better than high-density lipoprotein cholesterol levels and are favorably affected by Simvastatin treatment. Circulation, 108:2751-6.

Barter PJ, Baker PW, Rye KA. 2002. Effect of high-density lipoproteins on the expression of adhesion molecules in endothelial cells. Curr Opin Lipidol, 13:285-8.

Bedi US, Singh S, Syed A, et al. 2006. Coronary artery disease in South Asians: an emerging risk group. Cardiol Rev, 14:74-80.

Bhatnagar D, Anand IS, Durrington PN, et al. 2005. Coronary risk factors in people from the Indian subcontinent living in West London and their siblings in India. Lancet, 345:405-9.

Bhopal R, Fischbacher C, Vartiainen E. 2005. Predicted and observed cardiovascular disease in South Asians: application of FINRISK, Framingham and SCORE models to Newcastle Heart Project data. J Public Health, 27:93-100.

Bhopal R. 2002. Epidemic of cardiovascular disease in South Asians. BMJ, 16:324.

Boekholdt SM, Souverein OW, Tanck MW, et al. 2006. Common variants of multiple genes that control reverse cholesterol transport together explain only a minor part of the variation of HDL cholesterol levels. Clin Genet, 69:263-70. 
Calabresi L, Franceschini G. 1997. High density lipoprotein and coronary heart disease: Insights from mutations leading to low high density lipoprotein. Curr Opin Lipidol, 8:219-24.

Castelli WP, Garrison RJ, Wilson PW, et al. 1986. Incidence of coronary heart disease and lipoprotein cholesterol levels: The Framingham Study. JAMA, 256:2835-8.

Chhabra S, Narang R, Lakshmy R, et al. 2005. APO A-I 75 G to A substitution associated with severe forms of CAD, lower levels of HDL and Apo A-1 among Northern Indians. Disease Markers, 21:169-74.

D'Agostino RB Sr, Grundy S, Sullivan LM, et al. 2001. Validation of the Framingham coronary heart disease prediction scores: results of a multiple ethnic groups investigation. JAMA, 286:180-7.

Dodani S, Dong Y, Zhu H, et al. 2008a. Can novel Apo A-I polymorphisms be responsible for low HDL in South Asians. Indian J Hum Genetics, In press.

Dodani S, Kaur R, Mohammad N, et al. 2008b. Can dysfunctional HDL explain excess risk of coronary artery diseases in South Asian immigrants? Int J Cardiol, Feb 4. Epub ahead of print.

Downs JR, Clearfield M, Weis S, et al. 1998. Primary prevention of acute coronary events with lovastatin in men and women with average cholesterol levels: results of AFCAPS/TexCAPS. Air Force/Texas Coronary Atherosclerosis Prevention Study. JAMA, 279:1615-22.

Enas EA, Garg A, Davidson MA, et al. 1996. Coronary heart disease and its risk factors in first-generation immigrant Asian Indians to the United States of America. Indian Heart J, 48:343-53.

Enas EA, Yusuf S, Mehta J, et al. 1992. Prevalence of coronary artery disease in Asian Indians. Am J Cardiol, 70:945-9.

Enas EA. 2000. Coronary artery disease epidemic in Indians: a cause for alarm and call for action. J Indian Med Assoc, 98:694-5.

[NCEP ATP III] Expert Panel on Detection, Evaluation, and Treatment of High Blood Cholesterol in Adults. 2001. Executive summary of the third report of the National Cholesterol Education Program (NCEP) Expert Panel on Detection, Evaluation, and Treatment of High Blood Cholesterol in Adults (Adult Treatment Panel III). JAMA, 285:2486-97.

Fogelman AM. 2004. When good cholesterol goes bad. Nat Med, 10:902-3.

Forouhi N, McKeigue P. 1997. How far can risk factors account for excess coronary mortality in South Asians? Can J Cardiol, 13:47B.

Forouhi NG, Sattar N, Tillin T, et al. 2006. Do known risk factors explain the higher coronary heart disease mortality in South Asian compared with European men? Prospective follow-up of the Southall and Brent studies. Diabetologia, 49:2580-8.

Forouhi NG, Sattar N. 2006. CVD risk factors and ethnicity - a homogeneous relationship? Atheroscler, 7:11-9.

Frank PG Marcel YL. 2000. Apolipoprotein A-I: structure - function relationships. J Lipid Res, 41:853-72.

Gordon DJ, Probstfield JL, Garrison RJ, et al. 1989. High-density lipoprotein cholesterol and cardiovascular disease. Four prospective American studies. Circulation, 79:8-15.

Goyal A, Yusuf S. 2006. The burden of cardiovascular disease in the Indian subcontinent. Indian J Med Res, 124:235-44.

Graham A, Hassall DJ, Rafique S. 1997. Evidence for a paraoxonaseindependent inhibition of low-density lipoprotein oxidation by highdensity lipoprotein. Atherosclerosis, 135:193-204

Grundy SM. 2002. Obesity, metabolic syndrome, and coronary atherosclerosis. Circulation, 105:2696-8.

Guettier JM, Georgopoulos A, Tsai MY, et al. 2005. Polymorphisms in the fatty acid-binding protein 2 and apolipoprotein C-III genes are associated with the metabolic syndrome and dyslipidemia in a South Indian population. $J$ Clin Endocrinol Metab, 90:1705-11.

[HPS] Heart Protection Study Collaborative Group. 2002. MRC/BHF Heart Protection Study of cholesterol lowering with Simvastatin in 20,536 high-risk individuals: a randomized placebo-controlled trial. Lancet, 360:7-22.

Hoogeveen RC, Gambhir JK, Gambhir DS, et al. 2001. Evaluation of Lp[a] and other independent risk factors for CHD in Asian Indians and their USA counterparts. J Lipid Res, 42:631-8.
Hovingh GK, Brownlie A, Bisoendial RJ, et al. 2004. A novel apoA-I mutation (L178P) leads to endothelial dysfunction, increased arterial wall thickness, and premature coronary artery disease. $\mathrm{J} \mathrm{Am} \mathrm{Coll} \mathrm{Cardiol,}$ 44:1429-35.

Islam MS, Raitakari OT, Juonala M, et al. 2005. Apolipoprotein A-I/C-III/A-IV SstI and apolipoprotein B XbaI polymorphisms and their association with carotid artery intima-media thickness in the Finnish population. The Cardiovascular Risk in Young Finnish Study. Atherosclerosis, 180:79-86.

Jadhav UM, Kadam NN. 2004. Apolipoproteins: correlation with carotid intima media thickness and coronary artery disease. J Assoc Physicians India, 52:370-5.

Joshi P, Islam S, Pais P, et al. 2007. Risk factors for early myocardial infarction in South Asians compared with individuals in other countries. JAMA, 297:286-94.

Kalhan R, Puthawala K, Agarwal S, et al. 2001. Altered lipid profile, leptin, insulin, and anthropometry in offspring of South Asian immigrants in the United States. Metabolism, 50:1197-202.

Karathanasis SK. 1985. Apolipoprotein multigene family: tandem organization of human apolipoprotein AI, CIII, and AIV genes. Proc Natl Acad Sci U S A, 82:6374-8.

Klatsky AL, Tekawa I, Armstrong MA, et al. 1994. The risk of hospitalization for ischemic heart disease among Asian Americans in northern California. Am J Public Health, 84:1672-5.

Kobayashi KY, Yanagi H, Fukayama H, et al. 1998. Frequent occurrence of hypoalphalipoproteinemia due to mutant apolipoprotein A-I gene in the population: a population-based survey. Hum Mol Genet, 8:331-6.

Koistinen MJ, Huikuri HV, Korhonen UR, et al. 1994. Asymptomatic coronary artery disease in diabetes: relation to common risk factors, lipoproteins, apolipoproteins and apo E polymorphism. Acta Diabetol, $31: 210-14$.

Kottke TE, Thomas RJ, Lopez-Jimenez F, et al. 2006. CardioVision 2020: Program acceptance and progress after 4 years. Am J Prev Med, 30:137-43.

Kumar AP, Reynolds WF. 2005. Statins down regulate myeloperoxidase gene expression in macrophages. Biochem Biophys Res Commun, 331:442-51.

Luepker RV, Arnett DK, Jacobs JR, et al. 2006. Trends in blood pressure, hypertension control, and stroke mortality: the Minnesota Heart Survey. Am J Med, 119:42-9.

Lusis AJ, Fogelman AM, Fonarow GC, et al. 2004. Genetic basis of atherosclerosis: part II: clinical implications. Circulation, 110:2066-71.

Mackness MI, Arrol S, Abbott C, et al. 1993. Protection of low-density lipoprotein against oxidative modification by high-density lipoprotein associated paraoxonase. Atherosclerosis, 104:129-35.

Marathe GK, Zimmerman GA, McIntyre TM. 2003. Platelet-activating factor acetylhydrolase, and not paraoxonase-1 is the oxidized phospholipid hydrolase of high density lipoprotein particles. $J$ Biol Chem, 278:3937-47.

McGovern PG, Pankow JS, Shahar E, et al. 1996. Recent trends in acute coronary heart disease-mortality, morbidity, medical care, and risk factors. The Minnesota Heart Survey Investigators. $N$ Engl $J$ Med, 334:884-90.

Mohan V, Deepa R, Shanthi Rani SS, et al. 2001. Chennai Urban Population Study (CUPS No.5). Prevalence of coronary artery disease and its relationship to lipids in a selected population in South India. The Chennai Urban Population Study (CUPS No. 5). J Am Coll Cardiol, 38:682-7.

Moll PP, Michel VV, Weidman WH, et al. 1989. Genetic determination of plasma apolipoprotein A-I in a population based sample. Am J Hum Genet, 44:24-39.

Navab M, Anantharamaiah GM, Reddy ST, et al. 2004. Human apolipoprotein A-I and A-I mimetic peptides: potential for atherosclerosis reversal. Curr Opin Lipidol, 15:645-9.

Navab M, Anantharamaiah GM, Reddy ST, et al. 2006. Mechanisms of disease: proatherogenic HDL - an evolving field. Nat Clin Pract Endocrinol Metab, 2:504-11. 
Navab M, Ananthramaiah GM, Reddy ST. 2005. The double jeopardy of HDL. Ann Med, 37:173-8.

Navab M, Berliner JA, Subbanagounder G. 2001. HDL and the inflammatory response induced by LDL-derived oxidized phospholipids. Arterioscler Thromb Vasc Biol, 21:880.

Navab M, Hama SY, Anantharamaiah GM, et al. 2000. Normal high density lipoprotein inhibits three steps in the formation of mildly oxidized low density lipoprotein: steps 2 and 3. J Lipid Res, 41:1495-508.

Navab M, Hama SY, Cooke CJ, et al. 2000. Normal high density lipoprotein inhibits three steps in the formation of mildly oxidized low density lipoprotein: step 1. J Lipid Res, 41:1481-94.

Navab M, Hama SY, Hough GP, et al. 2001. A cell-free assay for detecting HDL that is dysfunctional in preventing the formation of or inactivating oxidized phospholipids. J Lipid Res, 42:1308-17.

Nordlie MA, Wold LE, Kloner RA. 2005. Genetic contributors toward increased risk for ischemic heart disease. J Mol Cell Cardiol, 39:667-9.

Perez-Mendez O, Bruckert E, Franceschini G, et al. 2000. Metabolism of apolipoproteins $\mathrm{AI}$ and $\mathrm{AII}$ in subjects carrying similar apoAI mutations, apoAI Milano and apoAI Paris. Atherosclerosis, 148:317.

Petersen S, Peto V, Rayner M. 2004. Coronary heart disease statistics, British Heart Foundation, London (2004) [online]. Accessed on February 10, 2008. URL: http://www.heartstats.org/datapage.asp?id $=1652$.

Pulkkinen A, Viitanen L, Kareinen A, et al. 2000. MspI polymorphism at +83 bp in intron 1 of the human apolipoprotein A1 gene is associated with elevated levels of HDL cholesterol and apolipoprotein A1 in nondiabetic subjects but not in type 2 diabetic patients with coronary heart disease. Diabetes Care, 23:791-5.

Raji A, Gerhard H, Warren M. 2004. Insulin resistance and vascular dysfunction in non diabetic Asian Indians. J Clin Endocrinol Metab, 9:3965-72.

Remaley AT, Stonik JA, Demosky SJ, et al. 2000. Apolipoprotein specificity for lipid efflux by the human ABCA1 transporter. Biochem Biophys Res Commun, 280:818-23.

Sacks FM, Pfeffer MA, Moye LA, et al. 1996. The effect of pravastatin on coronary events after myocardial infarction in patients with average cholesterol levels. N Engl J Med, 335:1001-9.

Sadaf A, Siddiqui S, Lestringant GG, et al. 2002. Apolipoprotein AI promoter variant in blood pressure determination. Clin Genet, 61:314-16.
[SSSG] Scandinavian Simvastatin Survival Group Study. 1994. Randomized trial of cholesterol lowering in 4444 patients with coronary heart disease: the Scandinavian Simvastatin Survival Study (4S). Lancet, 344:1383-9.

Shepherd J, Cobbe SM, Ford I, et al. 1995. Prevention of coronary heart disease with pravastatin in men with hypercholesterolemia. N Engl J Med, 333:1350-1.

Stampfer MJ, Sacks FM, Salvini S, et al. 1991. A prospective study of cholesterol, apolipoproteins, and the risk of myocardial infarction. N Engl J Med, 325:373-81.

Takahashi Y, Smith JD. 1999. Cholesterol efflux to apolipoprotein $\mathrm{AI}$ involves endocytosis and resecretion in a calcium-dependent pathway. Proc Natl Acad Sci U S A, 96:11358-63.

Tillin T, Forouhi N, Johnston DG. 2005. Metabolic syndrome and coronary heart disease in South Asians, African-Caribbeans and white Europeans: a UK population-based cross-sectional study. Diabetologia, 48:649-56.

Tulenko TN, Sumner AE. 2002. The physiology of lipoproteins. J Nucl Cardiol, 9:638-49.

Uppaluri CR. 2002. Heart disease and its related risk factors in Asian Indians. Ethn and Dis, 12:45-53.

Wang XL, Liu SX, McCredie RM, et al. 1996. Polymorphisms at the 5 prime-end of the apolipoprotein AI gene and severity of coronary artery disease. J Clin Invest, 98:372-7.

Watson KE, Ansell BJ, Watson AD, et al. 2007. HDL Function as a target of lipid-modifying therapy. Rev Cardiovasc Med, 8:1-8.

Williams B. 1995. Westernized Asians and cardiovascular disease: nature or nurture? Lancet, 345:401-2.

Williams DL, Connelly MA, Temel RE, et al. 1999. Scavenger receptor B1 and cholesterol trafficking. Curr Opin Lipidol, 10:329-39.

Yancey PG, Bortnick AE, Kellner-Weibel G, et al. 2003. Importance of different pathways of cellular cholesterol efflux. Arterioscler Thomb Vasc Biol, 23:712-19.

Zheng L, Nukuna B, Brennan ML, et al. 2004. Apolipoprotein A-I is a selective target for myeloperoxidase-catalyzed oxidation and functional impairment in subjects with cardiovascular disease. J Clin Invest, 114:529-41. 
